\title{
Non-Target Embolization of the Glans Penis During Prostatic Artery Embolization
}

\author{
Cameron James Parkin, ${ }^{凶 1,2,3}$ Cecile Pham, ${ }^{3,4}$ Amanda Chung, ${ }^{1,3,4,5}$ Stuart Menogue, ${ }^{4}$ Jules Catt, $, 6,7$ \\ Gavin Gottschalk, ${ }^{8}$ Cherie Wong, ${ }^{9}$ Venu Chalasani ${ }^{1}, 3,4,5$ \\ ${ }^{1}$ Department of Urology, Royal North Shore Hospital, Northern Sydney Local Health District, Australia ${ }^{2}$ School of Medicine, The University of Notre Dame, Sydney, \\ Australia ${ }^{3}$ North Shore Urology Research Group, Royal North Shore Hospital, NSW, Australia ${ }^{4}$ Department of Urology, Northern Beaches Hospital, NSW, Australia \\ ${ }^{5}$ Faculty of Health and Medicine, University of Sydney, Australia ${ }^{6}$ Department of Interventional Radiology, Liverpool Hospital, Australia ${ }^{7}$ Department of Radiology, \\ Prince of Wales Hospital, NSW, Australia ${ }^{8}$ Chatswood Dermatology Centre, NSW, Australia ${ }^{9}$ Department of Anatomical Pathology, Douglass Hanly Moir Pathology, \\ Macquarie Park, Australia
}

\section{Abstract}

Prostatic artery embolization is becoming increasingly popular in the management of benign prostatic hyperplasia, particularly for patients with significant comorbidities that make them poor candidates for either general anaesthesia or surgical intervention. Penile tip necrosis as a complication following prostatic artery embolization is exceedingly rare, with only 4 cases previously reported in the world literature. It occurs as a result of the embolization material passing into and occluding collateral arterial networks such as those supplying the glans penis. This paper identifies 3 further cases of penile tip necrosis, outlines its natural history, and proposes management strategies, so that clinicians can better identify and treat this condition.

Prostatic artery embolization (PAE) is an emerging interventional radiological procedure performed under local anaesthesia, which provides an alternative treatment for benign prostatic hyperplasia (BPH) in patients whose preference or medical comorbidities preclude either a general anaesthetic or surgical intervention $[1,2]$. PAE has been shown to improve lower urinary tract symptoms (LUTS), although not with the same efficacy as a transurethral resection of prostate (TURP) [3-5]. The largest case series reported is of 630 patients, in which $35 \%$ of patients reported an immediate improvement in symptoms, $82 \%$ had clinical success at medium-term follow-up (1 to 3 years), and $76 \%$ had clinical success at long-term follow-up (3 to 6.5 years)[3]. Reported complications following PAE include dysuria $(16.9 \%)$ and frequency (11.6\%), generally lasting no more than one week, and acute urinary retention (4.6\%)[6]. Penile tip necrosis as a complication is reported as being exceedingly rare $[3,4,6,7]$. This paper describes 3 cases of penile tip necrosis following PAE to enable clinicians to readily identify the complication and manage patients appropriately.

The first patient was a 63-year-old man with significant medical comorbidities with an American Society of Anesthesiologists Physical Status Classification System (ASA) score of 4, who presented to emergency in acute urinary retention. He had a history of obstructive symptoms and had trialled pharmacotherapy to limited effect, with an International Prostate Symptom Score (IPSS) of 17. He had undergone a successful PAE 3 years earlier, which resulted in an improvement in his urinary flow with a voided volume of $156 \mathrm{~mL}$, a peak flow rate of $15.1 \mathrm{~mL} / \mathrm{sec}$, but a slightly elevated post-void residual volume of $85 \mathrm{~mL}$. Following his re-presentation, he was referred back for consideration of repeating PAE. His prostate prior to the procedure measured $210 \mathrm{cc}$. A PAE via radial artery puncture was performed using $250 \mu \mathrm{m}$

\section{Key Words}

Prostatic artery embolization, penile tip necrosis, benign prostate hyperplasia, Embozene

\section{Competing Interests}

None declared

Patient Consent: Obtained.

\section{Article Information}

\author{
Received on May 19, 2021 \\ Accepted on July 17, 2021 \\ Soc Int Urol J.2021;2(5):323-326 \\ DOI: 10.48083/UCZ09737
}




\author{
Abbreviations \\ ASA American Society of Anaesthesiologists Physical \\ Status Classification System \\ $\mathrm{BPH}$ benign prostatic hyperplasia \\ IDC indwelling urethral catheter \\ IPSS International Prostate Symptom Score \\ LUTS lower urinary tract symptoms \\ PAE prostatic artery embolization \\ TURP transurethral resection of prostate
}

Embozene Microspheres. Pelvic angiography revealed the prostate arteries originating from the obturator arteries bilaterally. Small accessory pudendal arteries were identified originating from the obturator arteries, forming an arterial anastomosis between the prostate and penis (Figure 1). One week following the procedure, the patient noticed a rash developing over his glans penis. He was later referred to a dermatologist who noted full thickness epidermal necrosis and slough covering most of the glans (Figure 2a). The edge of the slough was lifted, and a punch biopsy performed. This revealed complete ulceration of the epithelium, inflammation and non-refractile spherical foreign bodies within the arterioles of the tissue, correlating with Embozene Microspheres used during the PAE procedure (Figure 2b). Three months following the PAE procedure, the necrotic areas over the glans had completely healed (Figure 2c).

\section{FIGURE 1.}

Pelvic angiography of a 63-year-old male (case 1) undergoing prostatic artery embolization, with a catheter placed in the left internal iliac artery. The left prostatic artery is derived from the left obturator artery which is also is supplying an accessory pudendal artery to the penis.

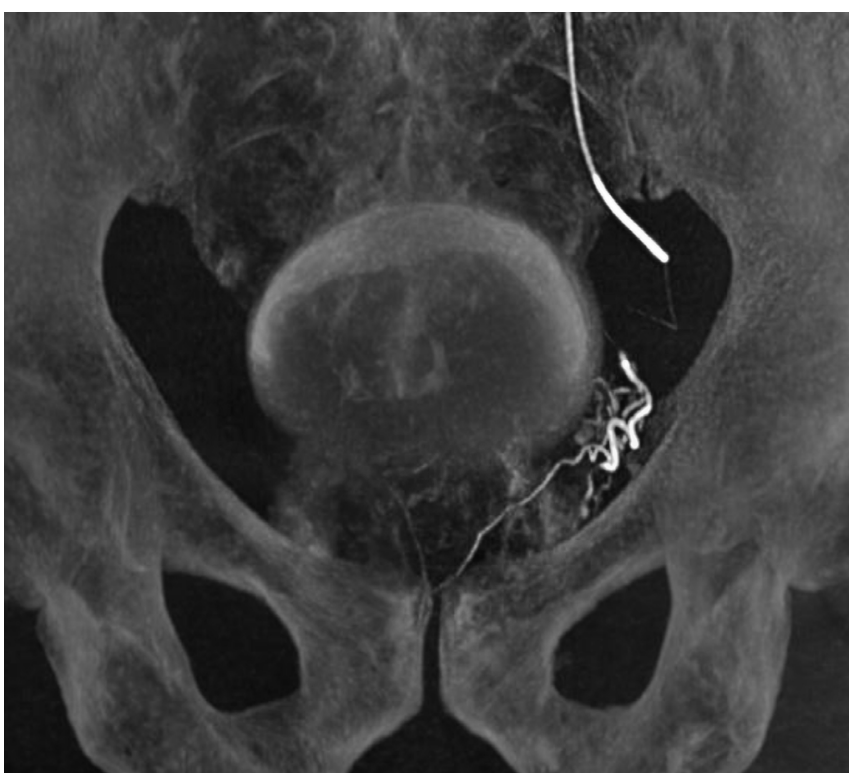

\section{FIGURE 2.}

Penile tip necrosis encountered following prostatic artery embolization: (A) A 63-year-old male (case 1) who developed penile tip necrosis 1 week following PAE; (B) Areas of necrosis over the glans were biopsied which revealed embolization of the Embozene Microspheres (circled) from the prostatic artery embolization within the vessels of the specimen, with surrounding inflammation and ulceration; (C) Three months following PAE, the areas of necrosis had completely healed; (D) An 84-year-old male (case 2) who developed penile tip necrosis day 7 postprocedure.

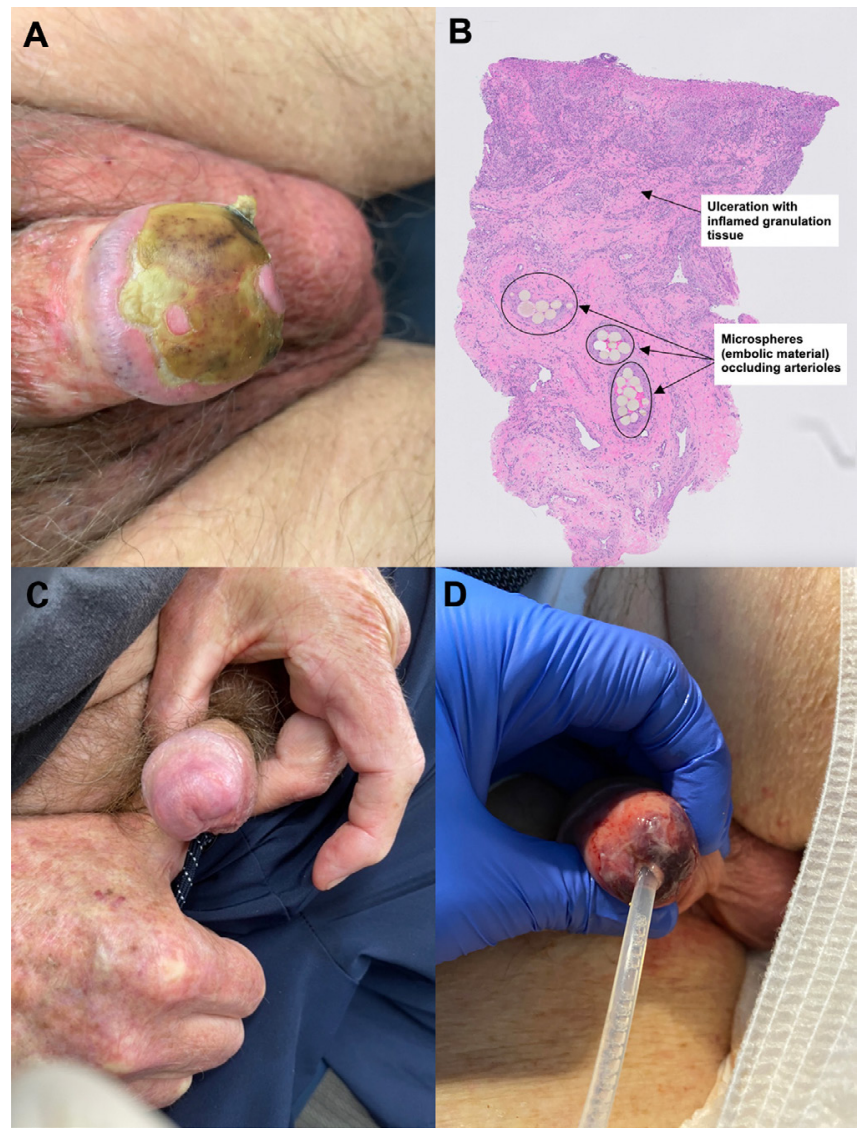

These had been managed with regular dressings and daily $2 \%$ lignocaine ointment. The patient subsequently passed a trial of void.

The second patient was an 84-year-old man, with an ASA score of 4, who was referred for management of $\mathrm{BPH}$ in the context of failed medical management. He had been experiencing worsening LUTS over 5 years and had been dependent on an indwelling urethral catheter (IDC). A pre-procedural computed tomography angiogram of the pelvis had revealed both the left and the right prostatic arteries arising from a gluteopudendal trifurcation. A PAE was undertaken via puncture of the right femoral artery. The left prostatic artery was embolized with $250 \mu \mathrm{m}$ Embozene Microspheres until complete stasis was achieved. No right-sided 
embolization was performed secondary to significant stenosis at the origin of the right prostatic artery. The day following the procedure, the patient developed penile tip pain and erythema. This was initially treated with simple analgesia and lignocaine $2 \%$ topical gel. Pain persisted and patchy areas of necrosis developed over the glans penis (Figure 2d). The patient was continued on regular tablet analgesia and clotrimazole $1 \%$ topical cream. By postoperative day 19 , the penile wounds had completely healed. The IDC was removed on postoperative day 60 , and the patient was able to void spontaneously.

The final patient was a 77-year-old man who initially presented to emergency in acute clot retention. A cystoscopy revealed the cause of haematuria to be a grossly enlarged trilobar prostate with a prominent vascularised median lobe. Ultrasound revealed a prostatic volume of $176 \mathrm{cc}$. He too had significant medical comorbidities and so was referred for PAE. Left radial artery access was obtained under local anaesthesia. Left internal iliac arteriography revealed the left prostatic artery branching from the middle rectal artery (Figure 3a). The left prostatic artery was catheterized and embolized with $250 \mu \mathrm{m}$ Embozene Microspheres. Right internal iliac arteriography revealed significant supply from an accessory pudendal artery (Figure $3 \mathrm{~b}$ ). The right prostatic artery was embolized with $250 \mu \mathrm{m}$ Embozene Microspheres distal to the origin of the accessory pudendal artery. The patient presented to the emergency department on day 7 postoperatively with worsening penile pain. He was noted to have an area of necrosis on the glans penis, just ventral to the urethral meatus, which was exquisitely tender. He was admitted and managed conservatively. At 1-month follow-up, he had ongoing penile pain and had developed ulceration at the urethral meatus. He was commenced on chloramphenicol $1 \%$ topical ointment twice daily. The IDC was removed at time of review, and he was able to void spontaneously. At 6-month follow-up, the patient had no pain, and the ulceration of the glans penis had healed.

Penile tip necrosis as a result of prostate artery embolization is rare, occurring in $<1 \%$ of cases [5]. Nontarget embolization refers to the process in which the passage of the particles used to occlude the prostate artery have passed into other arterial networks supplying pelvic organs[5]. Penile tip necrosis is a result of non-target embolization in which, during PAE, these particles have likely passed from the prostatic artery into the pudendal circulation supplying the penis. Patients who are vasculopaths with resultant abundant pelvic collaterization are at greater risk of non-target embolization[5]. The arterial supply of the penis as a result can be highly variable, where it can derive from the internal pudendal artery directly, from an accessory pudendal artery, or from a combination

\section{FIGURE 3.}

Three-dimensional reconstructions of a pelvic angiogram of a 77-year-old patient (case 3 ) undergoing prostatic artery embolization revealing selective catheterization of the left and right prostatic arteries (LPA \& RPA - white arrows). (A) The left prostatic artery originated from the middle rectal artery; (B) The right prostatic artery was identified to originate from an accessory pudendal artery (red arrow) which is also seen supplying the penis.

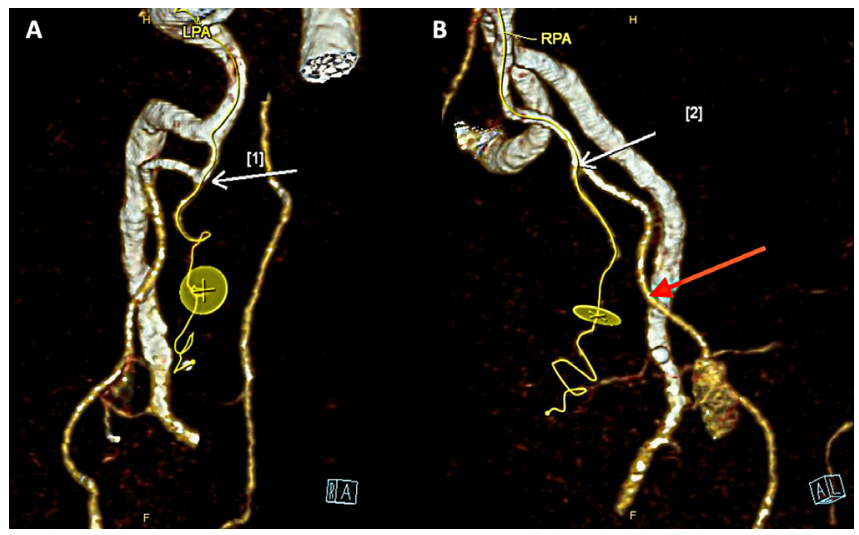

of the 2[8]. The accessory pudendal artery typically is a branch of the internal obturator artery or inferior vesical artery; the latter also supplies the prostatic arteries more proximally, thus forming an anastomosis between the prostate and the penis[8]. Patients who have accessory pudendal arteries are at higher risk of penile complications following PAE as a result [9]. Bilhim et al., in a retrospective study of 186 patients who underwent PAE for lower urinary tract symptoms, identified 9 patients (4.8\%) who developed small skin lesions on the glans penis following PAE[9]. These were likely small areas of necrosis, all of which had spontaneously healed within 1 month of the procedure[9]. The pelvic angiography of these cases revealed that all patients who suffered from this minor complication had prominent accessory pudendal arteries[9]. Thus, for patients in whom accessory pudendal arteries are identified, care must be taken with the embolization technique to clearly identify the arterial anatomy to minimize the occurrence of non-target embolization[9].

In all 3 cases discussed, $250 \mu \mathrm{m}$ Embozene Microspheres were used to occlude the prostatic arteries. Currently there is a wide variety in the size and composition of embolic agents available, with no consensus on a preferable agent. There is debate as to whether smaller particles, between $100 \mu \mathrm{m}$ and $300 \mu \mathrm{m}$, may predispose patients to nontarget embolization and recanalization of the arterial supply[10,11]. A small prospective randomised controlled trial has compared outcomes in 15 patients undergoing PAE who were embolized with smaller particles $(100 \mu \mathrm{m}$ to $300 \mu \mathrm{m}$ ) with outcomes in 15 patients embolized with larger particles $(300 \mu \mathrm{m}$ to $500 \mu \mathrm{m})[10]$. Functionally, 
both groups showed significant improvement in lower urinary tract symptoms as determined by mean IPSS scores. There were no major complications reported in either group, with no reports of penile tip necrosis. The patient cohort embolized with smaller agents did, however, report greater rates of complications, though these were not statistically significant $(P=0.066)$. These were mainly characterised by ejaculatory dysfunction and haematochezia.

As this is an uncommon occurrence following PAE, clinicians should still undertake a thorough clinical history and examination when approaching a new penile lesion. Penile lesions with similar appearance to that seen in these cases can be divided into benign, infective, inflammatory, and malignant aetiology. Penile biopsy should be reserved for those cases in which the diagnosis is unclear, or in which malignancy is suspected.

\section{References}

1. Huang M, Winoker J, Matlaga B, Allaf M, Koo K. Evidence-based analysis of online consumer information about prostate artery embolization for benign prostatic hyperplasia. Prostate Cancer Prostatic Dis.2021;24:106-113. DOl: 10.1038/s41391-020-0242-2

2. Kasivisvanathan V, Challacombe B, eds. The Big Prostate. 1st ed. Springer International Publishing;2017.

3. Pisco J, Bilhim T, Pinheiro LC, Fernandes L, Pereira J, Costa NV, et al. Medium- and long-term outcome of prostate artery embolization for patients with benign prostatic hyperplasia: results in 630 patients. J Vasc Interv Radiol.2016;27(8):1115-1122. D0I: 10.1016/j. jvir.2016.04.001

4. Ray A, Powell J, Speakman M, Longford NT, DasGupta R, Bryant T, et al. The UK ROPE Study: efficacy and safety of prostate artery embolization for benign prostatic hyperplasia. An observational study and propensity matched comparison with transurethral resection of the prostate. BJU Int.2018;122(2):270-282. DOI: 10.1111/bju.14249

5. Isaacson A, Bagla S, Raynor M, Yu H, eds. Prostatic Artery Embolization. 1st ed. Springer International Publishing; 2020.

6. Kably I, Richardson A, Naidu A, Sinha V, Bhatia S. Coil embolization of penile arterial collaterals during PAE efficacy and safety profile. J Vasc Interv Radiol.2018;29(4):S8.
Given its low incidence, there are no guidelines on the prevention and management of penile tip necrosis following PAE. Although certainly alarming in appearance, all cases reported have healed in time. Current literature suggests that in addition to local wound care, patients should be commenced on regular acetylsalicylic acid to reduce platelet aggregation at the site of necrosis and tadalafil to reduce cytokine mediated inflammation[5]. In the cases discussed, a stepwise analgesic regimen was followed, consisting of regular paracetamol, non-steroidal anti-inflammatories, and topical local anaesthesia in the form of $2 \%$ lignocaine. When concurrent infection is suspected, use of topical antimicrobials such as $1 \%$ chloramphenicol should be considered.

7. Kisilevzky N, Laudanna Neto C, Cividanes A. Ischemia of the glans penis following prostatic artery embolization. J Vasc Interv Radiol.2016;27(11):1745-1747.

8. Henry B, Pękala P, Vikse J, Sanna B, Skinningsrud B, Saganiak K, et al. Variations in the arterial blood supply to the penis and the accessory pudendal artery: a meta-analysis and review of implications in radical prostatectomy. J Urol.2017;198(2):345-353. DOI: 10.1016/j. juro.2017.01.080

9. Bilhim T. Endovascular resection of the prostate: how much is enough for prostate artery embolization? J Vasc Interv Radiol.2019;30(2):225227. DOI: 10.1016/j.jvir.2018.10.009

10. Goncalves O, Carnevale F, Moreira A, Antunes A, Rodrigues V, Srougi M. Comparative study using 100-300 versus $300-500 \mu$ m microspheres for symptomatic patients due to enlarged-BPH prostates. Cardiovasc Intervent Radiol.2016;39:1372-1378. DOI: 10.1007/s00270-016-1443-x

11. Bilhim T, Pisco J, Pereira J, Costa N, Fernandes L, Campos Pinheiro L, et al. Predictors of clinical outcome after prostate artery embolization with spherical and nonspherical polyvinyl alcohol particles in patients with benign prostatic hyperplasia. Radiology.2016;281(1):289-300. DOI: 10.1148/radiol.2016152292 